\title{
Local Intensity Variation Analysis for Iris Recognition
}

\author{
Li Ma, Tieniu Tan ${ }^{+}$, Dexin Zhang, Yunhong Wang \\ National Laboratory of Pattern Recognition, Institute of Automation, \\ Chinese Academy of Sciences, P.O. Box 2728, Beijing, P.R. China, 100080 \\ E-mails: \{lma,tnt,dxzhang,wangyh\}@nlpria.ac.cn
}

\begin{abstract}
As an emerging biometric for human identification, iris recognition has received increasing attention in recent years. This paper makes an attempt to reflect shape information of the iris by analyzing local intensity variations of an iris image. In our framework, a set of one-dimensional (1-D) intensity signals is constructed to contain the most important local variations of the original two-dimensional (2-D) iris image. Gaussian-Hermite moments of such intensity signals reflect to a large extent their various spatial modes and are used as distinguishing features. A resulting high dimensional feature vector is mapped into a low dimensional subspace using Fisher linear discriminant, and then the nearest center classifier based on cosine similarity measure is adopted for classification. Extensive experimental results show that the proposed method is effective and encouraging.
\end{abstract}

10

Keywords: Iris recognition, Gaussian-Hermite moments, Local intensity variations, Biometrics

\footnotetext{
${ }^{+}$Corresponding author. Tel: 86-10-62616658, Fax: 86-10-62551993, Email: tnt@nlpr.ia.ac.cn.
} 


\section{Introduction}

Biometric personal identification has been largely motivated by the increasing requirement for security in a networked society. Unlike traditional token-based or knowledge-based methods for personal identification, biometrics [1,2] employs various physiological or behavioral characteristics, such as fingerprints, face, facial thermograms, iris, retina, gait, palm-prints and hand geometry etc., to accurately identify each individual. As a physiological biometric, iris recognition aims to identify persons using iris characteristics of human eyes. Recently, iris recognition has received increasing attention due to its high reliability $[3,11,14]$.

The human iris, an annular part between the pupil (generally appearing black in an image) and the white sclera as shown in Figure 1(a), has an extraordinary structure and provides many interlacing minute characteristics such as freckles, coronas, stripes, furrows, crypts and so on. These visible characteristics, generally called the texture of the iris, are unique to each subject [5-14,30]. Individual differences that exist in the development of anatomical structures in the body result in such uniqueness. Some research work $[10,14,30-32]$ has also stated that the iris is essentially stable through a person's life. Furthermore, since the iris is an internal organ as well as externally visible, iris-based personal identification systems can be non-invasive to their users [10-14,31,32], which is greatly important for practical applications. All these desirable properties (i.e., uniqueness, stability and non-invasiveness) make iris recognition suitable for highly reliable personal identification.

Iris recognition relies greatly on how to accurately represent local details of the iris. Different from previous work on iris recognition [10-19], the proposed algorithm analyzes local intensity variations to reflect shape information of the iris. For an input 
approximate scale invariance, and then a set of 1-D intensity signals is generated to contain the most important local variations of the normalized 2-D iris image. Gaussian-Hermite moments, which use Gaussian-Hermite orthogonal polynomials as transform kernels, are adopted to characterize the details of such signals. Using Fisher linear discriminant, a resulting high dimensional feature vector is mapped into a low dimensional subspace, and then the nearest center classifier based on cosine similarity measure is utilized for recognition. Experimental results on an iris image database including 2255 images from 213 subjects, in terms of both absolute performance and comparative performance against several well-known iris recognition schemes, demonstrate the effectiveness and accuracy of the proposed method.

The primary contributions of this paper include:

1) A new attempt is made to represent the iris characteristics by analyzing local intensity variations of an iris image. Experimental results demonstrate that local intensity variations can effectively reflect shape information of the iris.

2) Detailed performance comparison among several well-known existing methods for iris recognition is conducted on a database of 2255 iris images.

The remainder of this paper is organized as follows. Section 2 describes related work. Section 3 introduces image preprocessing. Detailed descriptions of feature extraction and matching are respectively given in Section 4 and Section 5 . Experiments and results are reported in Section 6. Section 7 concludes this paper.

\section{Related work}

In 1987, Flom and Safir first proposed the concept of automated iris recognition [30], though an attempt to using the iris as an approach to human identification can be traced back to as early as 1885 [8]. By now, there have been some schemes for iris representation and matching in the recent literature [7,10-19,30-32]. Here, we have a 
brief look at the state of the art of iris recognition.

Unlike fingerprints, it is difficult to classify and localize apparent features in an iris image. From the viewpoint of feature extraction, existing iris recognition methods can be roughly divided into three major categories: the phase-based methods [10-12], the zero-crossing representation-based method [15] and the texture analysis-based methods $[13,14,16-19]$. Daugman [10-12] used multi-scale quadrature wavelets to extract texture phase structure information of the iris to generate a 2048-bit iriscode and compared the difference between a pair of iris representations by computing their Hamming distance via the XOR operator. Boles and Boashash [15] calculated zero-crossing representation of 1-D wavelet transform at various resolution levels of a virtual circle on an iris image to characterize the texture of the iris. Iris matching was based on two dissimilarity functions. Wildes et al. [13] represented the iris texture with a Laplacian pyramid constructed with four different resolution levels and used the normalized correlation to determine whether the input image and the model image are from the same class. Our previous work [17-19] adopted traditional texture analysis methods to capture the iris details. In [18], global texture features of the iris were extracted by means of well-known Gabor filters at different scales and orientations. Based on the experimental results and analysis obtained in [18], we further constructed a bank of spatial filters [19], whose kernels are suitable for iris recognition, to represent local texture features of the iris and thus achieved much better results. Lim et al. [16] decomposed an iris image into four levels using 2-D Haar wavelet transform and quantized the fourth-level high frequency information to form an 87-bit code. A modified competitive learning neural network (LVQ) was adopted for classification. It should be noted that all these algorithms are based on gray images, and color information is not used. The main reason is that the most 
discriminating information in recognition (i.e. variations of the iris texture) is the same in both gray and color images.

Great progress has been made in iris recognition. However, most existing methods for iris recognition generally used some small image sets for performance evaluation, and only the method by Daugman has been tested on a larger image database involving over 200 subjects $[3,11,12]$. Currently, there is also no quantitative comparison among these methods [10-19]. So we construct an iris image database including 2255 images from 213 subjects to compare their recognition performance and provide detailed discussions as well. Since the complex texture of the iris comprises a large number of interlacing small blocks such as freckles, coronas, stripes, furrows, crypts etc., a desirable representation method should be able to well characterize the shape of such blocks. In this paper, we make an attempt to analyze local intensity variations of an iris image to reflect shape information of the iris.

\section{Image preprocessing}

An iris image, as shown in Figure 1(a), contains not only the region of interest (iris) but also some irrelevant parts (e.g. eyelid, pupil etc.). A change in the camera-to-eye distance may also result in variations in the size of the same iris. Furthermore, the brightness is not uniformly distributed because of non-uniform illumination. Therefore, before feature extraction, the original image needs to be preprocessed to localize iris, normalize iris, and reduce the influence of the factors mentioned above. Such preprocessing is detailed in the following subsections.

\subsection{Iris localization}

The iris is an annular portion between the pupil (inner boundary) and the sclera (outer boundary). Both the inner boundary and the outer boundary of a typical iris can 
approximately be taken as circles. However, the two circles are usually not concentric [10]. We localize the iris using a simple but effective method:

1) Since the pupil is generally darker than its surroundings, the iris region in an image can approximately be found by projecting the image in the horizontal and vertical direction according to the following equation:

$$
\begin{aligned}
& X_{p}=\arg \min _{x}\left(\sum_{y} I(x, y)\right) \\
& Y_{p}=\arg \min _{y}\left(\sum_{x} I(x, y)\right)
\end{aligned}
$$

where $X_{p}$ and $Y_{p}$ denote the center coordinates of the pupil in the original image $I(x, y)$.

2) The exact parameters of these two circles are obtained by using edge detection (Canny operator in our experiments) and Hough transform in a rectangular region centered at the point $\left(X_{p}, Y_{p}\right)$ determined in the first step. Such processing results in a lower computational cost.

An example of iris localization is shown in Figure 1(b).

\subsection{Iris normalization}

Irises from different people may be captured in different size, and even for irises from the same eye, the size may change because of illumination variations and other factors (the pupil is very sensitive to lighting changes). Such elastic deformation in iris texture will influence the results of iris matching. For the purpose of achieving more accurate recognition results, it is necessary to compensate for such deformation. Here, we anti-clockwise unwrap the iris ring to a rectangular texture block with a fixed size $(64 \times 512$ in our experiments) by a linear mapping. The normalization can thus reduce to a certain extent the distortion of the iris caused by pupil movement. A normalized image is shown in Figure 1(c). 


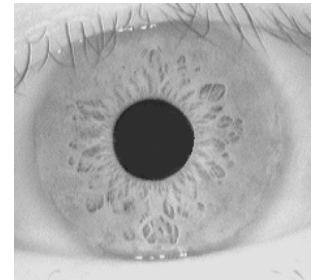

(a)

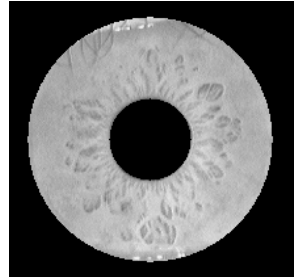

(b)

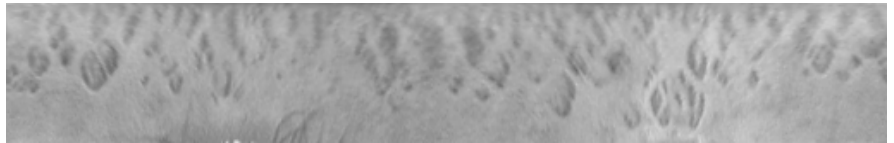

(c)

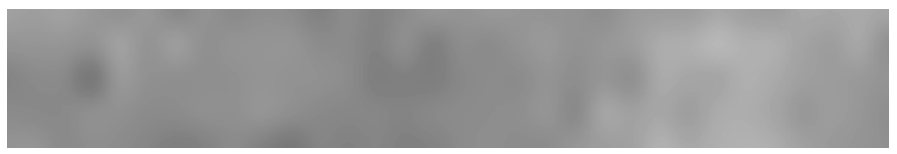

(d)

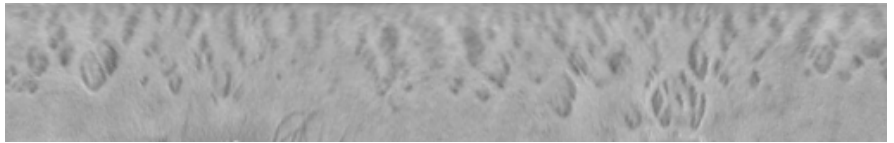

(e)

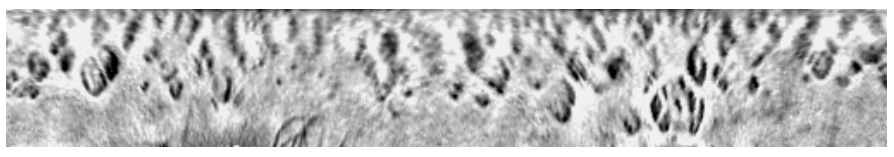

(f)

Figure 1. Iris image preprocessing

(a) Original image; (b) Localized image; (c) Normalized image;

(d) Estimated background illumination; (e) Lighting corrected image; (f) Enhanced image.

\subsection{Image enhancement}

The normalized iris image has still low contrast and may have non-uniform brightness caused by the position of light sources. All these may affect the subsequent feature extraction and matching. In order to obtain a more well-distributed texture image, we first approximate intensity variations across the whole image. The mean of each 16x16 small block constitutes a coarse estimate of the background illumination. This estimate is further expanded to the same size as the normalized image by bicubic interpolation. The estimated background illumination as shown in Figure 1(d) is subtracted from the normalized image to compensate for a variety of lighting 
conditions. Then we enhance the lighting corrected image by means of histogram equalization in each $32 \times 32$ region. Such processing compensates for non-uniform illumination, as well as improving the contrast of the image. Figure 1(f) shows the preprocessing result of an iris image, from which we can see that finer texture characteristics of the iris become clearer than that in Fig. 1(c).

\section{Feature extraction}

By intuitive observations about iris images, we find that the shape (such as freckles, coronas, stripes and furrows etc.) can be considered as an elementary component of the iris texture. We thus anticipate that shape information provides discriminating features in iris recognition. As we know, the shape is generally characterized by the object contours (namely image edges). However, it is difficult to well segment the irregular iris blocks of a very small size in gray images. Such irregular blocks cause noticeable local intensity variations in iris images. Therefore, we approximately reflect shape information of the iris characteristics by analyzing the resulting local variations in the iris image. A normalized image is first decomposed into a set of 1-D intensity signals which retain most local variations of the iris, and then important and meaningful features are extracted from such signals. The moment-based method has been widely used to represent local characteristics of images in pattern recognition and image processing [20-24]. Here, Gaussian-Hermite moments are adopted to characterize local variations of the intensity signals.

\subsection{Generation of 1-D intensity signals}

Generally, local details of the iris spread along the radial direction in the original image corresponding to the vertical direction in the normalized image (see Figure 2 and 4). Therefore, information density in the angular direction corresponding to the horizontal direction in the normalized image is much higher than that in other 
directions $[11,19]$. In addition, since our basic idea is to reflect shape information of the randomly-distributed blocks by analyzing local variations in the iris image, it is unnecessary to capture local variations in every line of the iris image for recognition. Bearing these two points in mind, we decompose the 2-D normalized image into a set of 1-D intensity signals by use of the following equation:

$$
\begin{aligned}
& S_{i}=\frac{1}{M} \sum_{j=1}^{M} I_{(i-1)^{*} M+j} \quad i=1,2, \ldots N \\
& I=\left(\begin{array}{l}
I_{1} \\
\vdots \\
I_{x} \\
I_{K}
\end{array}\right)=\left(I_{1}^{T}, \cdots I_{x}^{T}, \cdots I_{K}^{T}\right)^{T}
\end{aligned}
$$

where $I_{x}$ denotes gray values of the $x$ th row in the normalized image $I$ of $K \times L(64 \times 512$ in our experiments), $M$ is the number of rows used to form a signal $S_{i}$, and $N$ is the total number of 1-D signals. In essence, each signal is the average of $M$ successively horizontal scan lines which reflect local variations of an object along the horizontal direction. A set of such signals should contain the majority of local variations of the iris. Additionally, such processing reduces the computational cost required for the subsequent feature representation. The recognition rate of the proposed algorithm can be regulated by changing the constant $M$. A small $M$ leads to a large set of signals, which results in analyzing the iris characteristics in more detail, and thus increases the recognition accuracy. On the contrary, a large $M$ may incur a higher computational efficiency but a lower recognition accuracy.

In experiments, we find that the iris region closer to the pupil provides the most discriminating information for recognition (see Fig. 2) and is also rarely occluded by eyelids and eyelashes. So we extract features only in the region closer to the pupil. The region of interest (ROI) shown as the section above the dotted line in Fig. 2 takes up about $80 \%$ of the normalized image. 


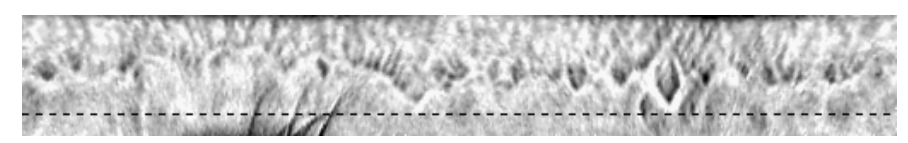

(a)

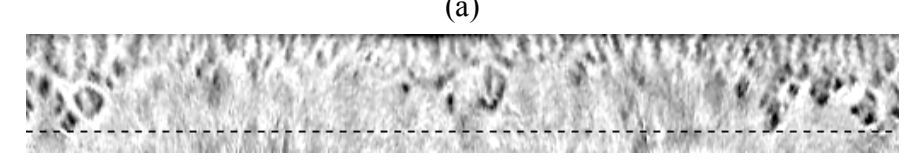

(b)

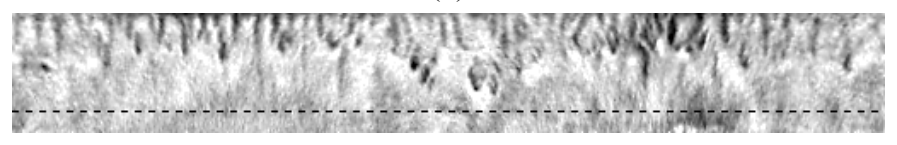

(c)

Figure 2. Illustration of the ROIs

\subsection{Gaussian-Hermite moments}

Moments have been widely used in pattern recognition and image processing, especially in various shape-based applications. More recently, the orthogonal moment based method has been one of the active research topics in shape analysis. Unlike commonly used geometric moments, orthogonal moments use orthogonal polynomial functions as transform kernels, which produces minimal information redundancy. The detailed study on the different moments and their behavior evaluation may be found in $[21,24]$. Here, Gaussian-Hermite moments are used for feature extraction due to their mathematical orthogonality and effectiveness for characterizing local details of the signal $[23,24]$. The $n$th order 1-D Gaussian-Hermite moment $M_{n}(x)$ of a signal $S(x)$ is defined as:

$$
M_{n}(x)=\int_{-\infty}^{\infty} K_{n}(t) S(x+t) d t \quad n=0,1,2, \ldots
$$

15 with

$$
\begin{aligned}
& K_{n}(t)=g(t, \sigma) H_{n}(t / \sigma) \\
& H_{n}(t)=(-1)^{n} \exp \left(t^{2}\right) \frac{d^{n} \exp \left(-t^{2}\right)}{d t^{n}}
\end{aligned}
$$

where $g(t, \sigma)$ is a Gaussian function, $H_{n}(t)$ is a Hermite polynomial function of order $n$, and the kernel $K_{n}(t)$ is a product of these two functions. Figure 3 shows the spatial responses of the Gaussian-Hermite moment kernels of different orders and their corresponding Fourier transforms. 


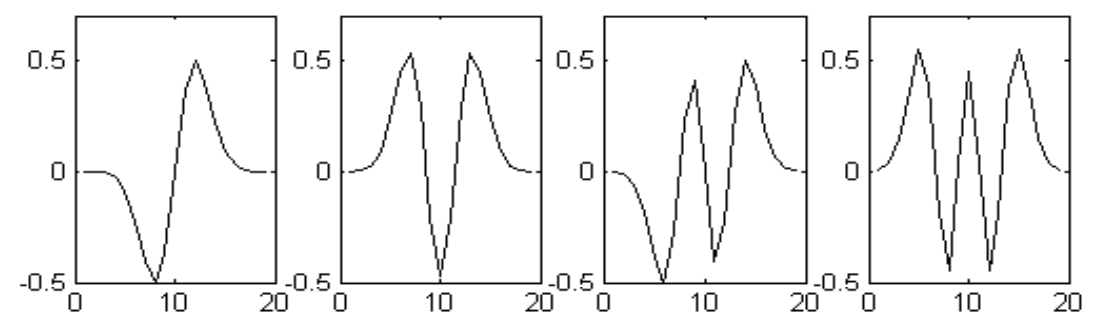

(a)
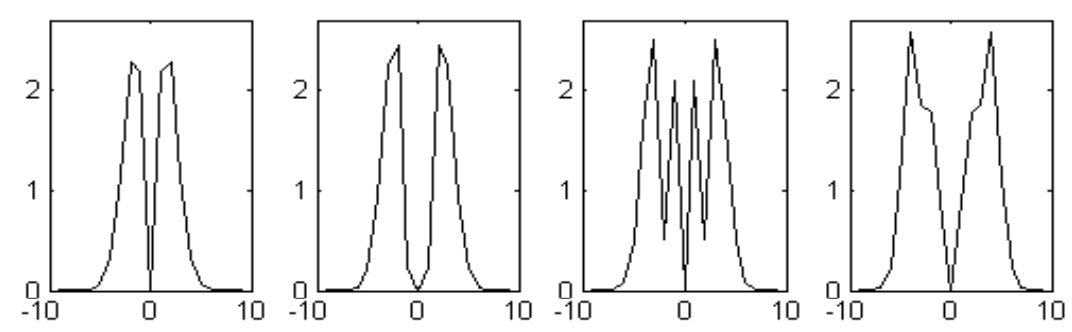

(b)

Figure 3. Gaussian-Hermite moment kernels:

(a) the spatial responses of the Gaussian-Hermite moment kernels, order 1 to 4;

(b) the corresponding Fourier spectra of (a).

From Figure 3, we can see that with the increase of the order of the moment kernels, oscillations of the moment kernels also increase, implying that the moment kernels of different orders correspond to different spatial modes. In fact, Gaussian-Hermite moments are linear combinations of the derivatives of the signal filtered by a Gaussian filter [24]. As is well known, the derivatives are important features for representing local properties of a signal. In image processing, we often use the derivatives of different orders to effectively characterize the image, but how to combine them is still a difficult problem. Gaussian-Hermite moments provide an approach to constructing orthogonal features from different derivatives. Here, our purpose is to analyze local variations of the resulting intensity signals. Gaussian-Hermite moments can well represent different spatial modes and are thus capable of effectively characterizing the differences between 1-D intensity signals. Moreover, from the viewpoint of spectral analysis, each moment kernel is somewhat similar to a bandpass filter. The higher the moment kernel's order is, the higher its spatial frequency. This means that Gaussian-Hermite moments capture signal features 
over a broad frequency range. These desirable characteristics exhibited by Gaussian-Hermite moments make them a suitable choice for analyzing local variations of the intensity signals.

\subsection{Feature vector}

For each signal $S_{i}$ described in Section 4.1, we can calculate its Gaussian-Hermite moment $M_{i, n}$ of order $n$ according to Equation (3). In our experiments, we generate 10 intensity signals from the ROI, $i \in\{1, \ldots, 10\}$, and use 4 different order Gaussian-Hermite moments, $n \in\{1,2,3,4\}$. In addition, the space constant of the Gaussian function in Equation (4) affects the shape of the Gaussian-Hermite moment kernels. In the experiments, it is set to 2.5. Since the outputs $M_{i, n}$ denote different local features derived using different moment kernels, we concatenate all these features together to form an integrated feature vector,

$$
V=\left[M_{1,1}, M_{1,2}, \ldots M_{10,3}, M_{10,4}\right]^{T}
$$

where $T$ is the transpose operator. Since the length of each intensity signal is 512 , the feature vector $V$ includes $20480(512 \times 10 \times 4)$ components. To reduce the space dimension and the subsequent computational complexity, we can 'downsample' each moment $M_{i, n}$ by a factor $d$ before the concatenation. Here, downsampling means replacing $d$ successive feature elements by their average. So, the downsampled feature vector $V^{d}$ can be rewritten as follows:

$$
V^{d}=\left[M_{1,1}{ }^{d}, M_{1,2}{ }^{d}, \ldots M_{10,3}{ }^{d}, M_{10,4}{ }^{d}\right]^{T}
$$

Equation (6) shows that the integrated feature vector takes all the outputs $M_{i, n}{ }^{d}$ as discriminating information.

\subsection{Invariance}

It is desirable to obtain an iris representation invariant to translation, scale, and rotation. Invariance to translation is intrinsic to our algorithm since feature extraction 
is based on a set of intensity signals instead of the original image. To achieve approximate scale invariance, we normalize an input image to a rectangular block of a fixed size. We can also provide approximate rotation invariance by downsampling each moment $M_{i, n}$ derived in Section 4.3 at different initial position. That is, each moment $M_{i, n}$ is circularly shifted before downsampling. In our experiments, the shift values are $-12,-8,-4,0,4,8,12$, which approximately correspond to rotate the original iris by $-9^{\circ},-6^{\circ},-3^{\circ}, 0,3^{\circ}, 6^{\circ}, 9^{\circ}$, respectively (Note that in practical applications, it is unlikely to have very large rotation angles as the user's face is usually nearly upright). We thus define seven templates which respectively denote the seven rotation angles for each iris class in the database. This brings the extra computational expense. Since the template generation is an off-line process, it is not a very serious problem. When matching the input feature vector with the templates of a class, the minimum of the seven scores is taken as the final matching distance.

\section{Matching}

By feature extraction, an iris image can be represented as a high dimensional feature vector depending on the downsampling factor $d$. To reduce the computational cost and improve the classification accuracy, Fisher linear discriminant is first used to generate a new feature vector with salient information of the original feature vector, and then the nearest center classifier is adopted for classification in a low dimensional feature subspace.

Two popular methods for dimensionality reduction are principal component analysis (PCA) and Fisher linear discriminant (FLD). Compared with the PCA, the FLD not only utilizes information of all samples but also shows interest in the underlying structure of each class. In general, the latter can be expected to outperform the former [26,27]. Fisher linear discriminant searches for projected vectors that best 
discriminate different classes in terms of maximizing the ratio of between-class to within-class scatter, which can be described by the following equation:

$$
\begin{aligned}
& W=\arg \max _{w} \frac{\left|W^{T} S_{B} W\right|}{\left|W^{T} S_{W} W\right|}=\left[\begin{array}{llll}
w_{1} & w_{2} & \cdots & w_{m}
\end{array}\right] \\
& S_{B}=\sum_{i=1}^{c}\left(\mu_{i}-\mu\right)\left(\mu_{i}-\mu\right)^{T} \\
& S_{W}=\sum_{i=1}^{c} \sum_{j=1}^{N_{i}}\left(x_{j}^{i}-\mu_{i}\right)\left(x_{j}^{i}-\mu_{i}\right)^{T}
\end{aligned}
$$

where $c$ is the total number of classes, $\mu$ is the mean of all samples, $\mu_{i}$ is the mean of the $i$ th class, $N_{i}$ is the number of samples of the $i$ th class, $x_{j}^{i}$ is the $j$ th sample of the $i$ th class, $S_{B}$ is the between-class scatter matrix, and $S_{W}$ is the within-class scatter matrix. In our experiments, an enhanced Fisher discrimination model (EFM) is utilized for the solution to the optimal projective matrix $W$. The EFM method [28] improves the generalization capability of Fisher linear discriminant using a more effective numerical solution approach. Further details of Fisher linear discriminant may be found in [25-29].

The new feature vector, $f$, is defined as follows:

$$
f=W^{T} V^{d}
$$

where $V^{d}$ is the original feature vector derived in Section 4.3. The proposed algorithm makes use of the nearest center classifier defined in Equation (9) for classification in a low dimensional feature subspace constructed by the optimal projective matrix $W$.

$$
\begin{aligned}
& j=\arg \min _{1 \leq i \leq c} d\left(f, f_{i}\right) \\
& d\left(f, f_{i}\right)=1-\frac{f^{T} f_{i}}{\|f\|\left\|f_{i}\right\|}
\end{aligned}
$$

where $f$ is the feature vector of an unknown sample, $f_{i}$ is the feature vector of the $i$ th class, $c$ is the total number of classes, $\|\bullet\|$ denotes the norm operator, and $d\left(f, f_{i}\right)$ is cosine similarity measure. The feature vector, $f$, is classified into the $j$ th class, the closest mean, using the similarity measure $d\left(f, f_{i}\right)$. 


\section{Experimental results}

Extensive experiments on a reasonably sized image database are performed to evaluate the effectiveness and accuracy of the proposed method. The experiments are completed in two modes: identification (one-to-many matching) and verification (one-to-one matching). In identification mode, for a test sample, the algorithm makes a one-to-many search of the entire database to find a template most like the test sample. If the test sample and the found template are from the same class, this is a correct recognition. Therefore, in identification mode, the algorithm can be measured by Correct Recognition Rate (CRR), the ratio of the number of samples being correctly classified to the total number of test samples. In verification mode, assuming that a test sample is from a specified subject, a one-to-one comparison is made to verify whether the test sample is from the specified subject. Such comparisons result in two independent error rates, False Match Rate (FMR) and False Non-Match Rate (FNMR). The FMR (sometimes called false positive rate) is the probability that a test sample of an imposter is falsely declared to match a template of an authorized subject and the FNMR (sometimes called false negative rate) is the probability that a test sample of an authorized subject is falsely declared not to match his template. By adjusting a matching threshold, a Receiver Operating Characteristic (ROC) curve [3,4] can be created. The ROC curve is a plot of genuine match rate (1-FNMR) against false match rate for all possible matching thresholds and shows the overall performance of an algorithm. The ideal ROC curve is a step function at the zero false match rate. Two commonly used performance measures derived from the ROC curve are the area under the ROC curve (denoted as AUC or Az) and Equal Error Rate (EER). The Az reflects how well the intra-class and inter-class distributions can be distinguished and generally ranges from 0.5 to 1 . A value of 0.5 implies that the intra-class and inter-class distributions are the exactly same (hence completely 
inseparable). In the ideal case, the Az should be 1 (namely both the FMR and FNMR are zero), indicating that the intra-class and inter-class distributions are disjoint. The EER is the point where the false match rate and the false non-match rate are equal in value. The smaller the EER is, the better the algorithm. In our experiments, the measures described above are used for performance evaluation. The following subsections detail the experiments and results.

\subsection{Image database}

Unlike fingerprint and face, there is no common iris database of a reasonable size for algorithm evaluation. Therefore, we collect a large number of iris images using a homemade digital optical sensor to form a database named CASIA Iris Database. The database includes 2255 iris images from 306 different eyes (hence 306 different classes) of 213 subjects. The images are acquired during different stages and the time interval between two collections is at least one month, which provides a challenge to our algorithm. To the best of our knowledge, this is currently the largest iris database available in the public domain. The profile of the database is shown in Table 1. The subjects consist of 203 members of the CAS Institute of Automation and 10 visiting students from Europe.

Table 1. CASIA Iris Database

\begin{tabular}{|c|c|c|c|c|c|}
\hline \multicolumn{2}{|c|}{ Subjects } & \multicolumn{2}{c|}{ Age and Gender } & Time Interval & Environment \\
\hline Chinese & $95.3 \%$ & Age $<25$ & $41 \%$ & & \\
\cline { 3 - 4 } & $25<=$ Age $<50$ & $55 \%$ & $\begin{array}{c}\text { At lease one month } \\
\text { between two } \\
\text { capture stages }\end{array}$ & $\begin{array}{c}\text { Normal office } \\
\text { conditions } \\
\text { (indoor) }\end{array}$ \\
\hline Others & $4.7 \%$ & Age $>=50$ & $4 \%$ & $7: 3$ & \\
\cline { 3 - 4 } & Male : Female & $7: 3$ & \\
\hline
\end{tabular}

The homemade sensor works in PAL mode (i.e., 25 frames/second) and provides near infrared illumination under which the iris exhibits more abundant texture features. The subject needs to position himself about $4 \mathrm{~cm}$ in front of the sensor to obtain a clear iris image. Moreover, a surface-coated semi-transparent mirror is placed in front of 
the lens so that a person can see and keep his eye in the center of the sensor. The captured iris images are 8-bit gray images with a resolution of 320x280. In general, the diameter of the iris in an image from our database is greater than 200 pixels. This makes sure that there is enough texture information for reliable iris recognition. The CASIA Iris Database includes two main parts. One is our earlier image set [18] containing 500 images from 25 different subjects. Each individual provides 20 images (10 for each eye). In the first stage, five images of each eye are acquired. Four weeks later, five more images of each eye are taken. The other part contains 1755 images from 188 subjects which are captured in three different stages. The images form 256 iris classes (Note that not every individual provides iris images of both eyes, but at least 5 images for each eye). The total number of iris classes is thus $306(2 \times 25+256)$. Some samples from the CASIA Iris Database are shown in Figure 4. For each iris class, we choose three samples from images taken in the first stage for training and all samples captured at other stages serve as test samples. This is consistent with the widely accepted standard for biometrics algorithm testing [3,4] (Training images and testing images should be respectively captured at different stages). To satisfy this requirement, 100 images taken in the first stage are not used in the experiments. Therefore, for the CASIA Iris Database, there are 918 images for training and 1237 images for testing.
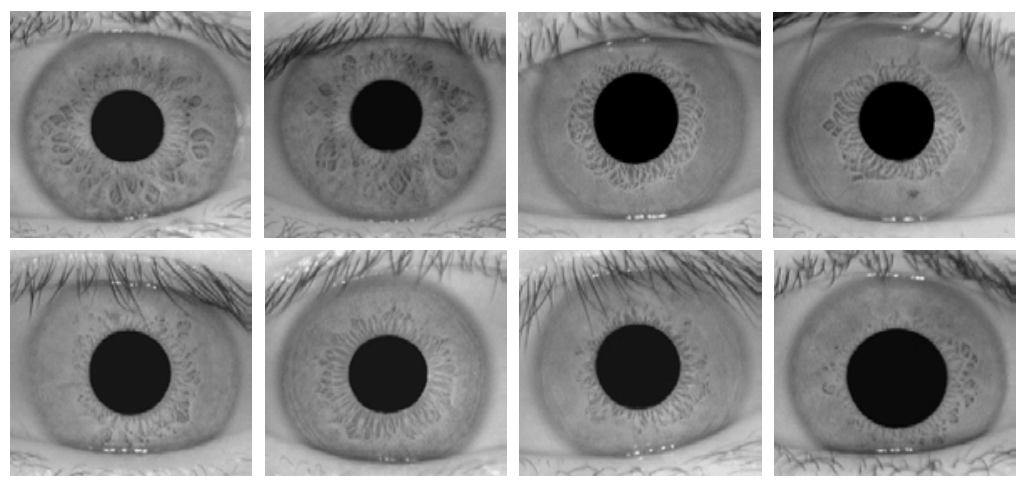

Figure 4. Iris samples from the CASIA Iris Database 


\subsection{Recognition results}

The downsampling factor $d$ defined in Eq. (6) has important effects on both the accuracy and the computational cost of the whole algorithm. In fact, it is a tradeoff between accuracy and speed. A large downsampling factor $d$ means a fast speed while a low accuracy. In contrast, a small downsampling factor $d$ results in a high accuracy but a slow speed. We thus carry out experiments on the integrated feature vector $V^{d}$ defined by Eq. (6) with different downsampling factors. From the recognition results shown in Table 2, we can see that the performance differences are not very significant when the downsampling factor is less than 32 , whereas when the downsampling factor is 64 , the recognition rate descends dramatically. This is because that the larger the downsampling factor is, the more information of the iris loses. We choose the downsampling factor of 16 in the subsequent experiments since it maintains a good compromise between accuracy and speed. Therefore, the dimensionality of the feature vector after downsampling is 1280 .

Table 2. Recognition results using different downsampling factors

\begin{tabular}{ccc}
\hline $\begin{array}{c}\text { Downsampling } \\
\text { factor }\end{array}$ & $\begin{array}{c}\text { Dimensionality } \\
\text { of features }\end{array}$ & Recognition rate (\%) \\
\hline 8 & 2560 & 99.68 \\
16 & 1280 & 99.52 \\
32 & 640 & 98.71 \\
64 & 320 & 86.98 \\
\hline
\end{tabular}

Figure 5 describes variations of the recognition rate with the increasing dimensionality of the reduced feature vector, from which we can find that with the increase of dimensionality of the reduced feature vector, the recognition rate also rapidly increases. However, when the dimensionality of the reduced feature vector is up to 120 or much higher, the recognition rate nearly stabilizes at a very high level of about $99.43 \%$. Particularly, the proposed method achieves $99.60 \%$ recognition rate 
using 160 features. As a result, we use only 160 features in the next series of experiments.

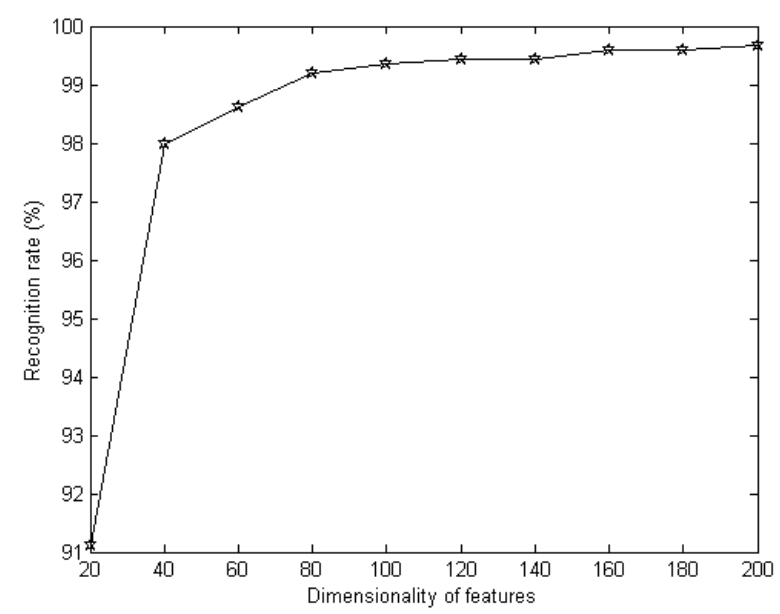

Figure 5. Recognition performance under different dimensionality of features

We test the proposed algorithm in two modes, namely identification and verification. In identification tests, an overall correct recognition rate of $99.60 \%$ is achieved. The verification results are shown in Figure 6 and Table 3. Figure 6 is the ROC curve of the proposed method on the CASIA Iris Database. Points on this curve denote all possible system operating states in different tradeoffs. To exhibit the possible operating states more clearly, the horizontal axis of Figure 6 is spread out using the logarithmic scale. The Az (the area under the ROC curve) is up to 0.9998, and the EER is only $0.29 \%$. These results are quite encouraging and indicate the high performance of the proposed method. Table 3 lists three typical operating states of the proposed method. In particular, if one and only one false match occurs in 100,000 trails, the false non-match rate is only $1.13 \%$. The experimental results demonstrate that the proposed iris representation is effective and local variations can well represent the differences between irises. 


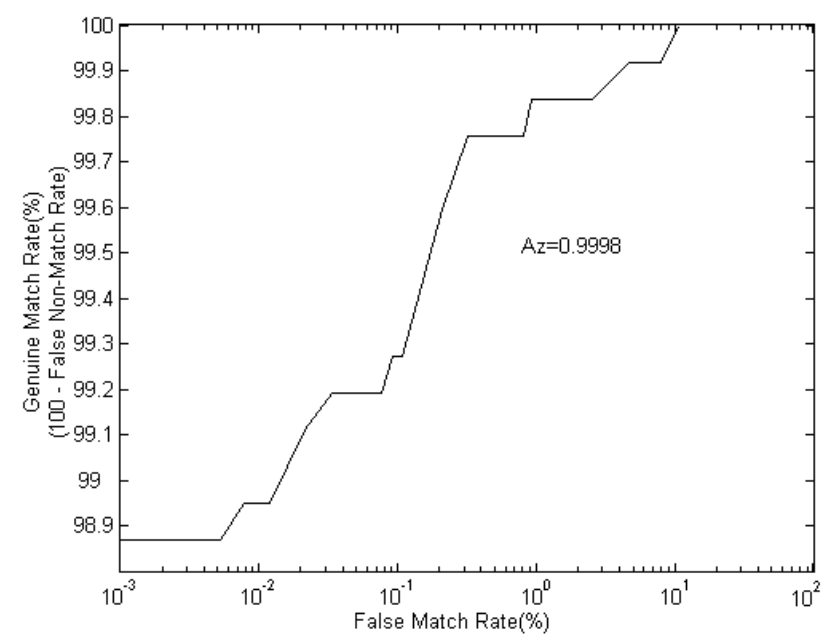

Figure 6. Verification results

Table 3. The typical operating states of the proposed method

\begin{tabular}{cc}
\hline False match rate $(\%)$ & False non-match rate $(\%)$ \\
\hline 0.001 & 1.13 \\
0.01 & 1.05 \\
0.1 & 0.65 \\
\hline
\end{tabular}

\subsection{Comparison and discussions}

Among existing methods for iris recognition, those proposed by Daugman [10-12], Wildes et al. [13], and Boles et al. [15] respectively are the best known. Moreover, they characterize the local details of the iris from different viewpoints, i.e. phase-based approach, texture analysis-based approach and zero-crossing representation method. To further prove the effectiveness of the proposed method, we make detailed comparison between the current method and the above three methods (and our previous work $[18,19]$ ) on the CASIA Iris Database in two modes (verification and identification). For the purpose of comparison, we implement the three methods according to the published papers [10-15,31,32] (We compare our experimental results of each algorithm with its published results and find that they are consistent. This verifies to a large extent the correctness of our implementations of 
other methods.). Because the method by Wildes et al. [13] only works in verification mode, we do not test its performance in identification mode. Table 4 and Figure 7 detail the experimental results.

Table 4. Comparison of CRRs, Azs and EERs

\begin{tabular}{cccc}
\hline Methods & $\begin{array}{c}\text { Correct recognition } \\
\text { rate (\%) }\end{array}$ & $\begin{array}{c}\text { Az (area under } \\
\text { the ROC curve) }\end{array}$ & $\begin{array}{c}\text { Equal error } \\
\text { rate }(\%)\end{array}$ \\
\hline Boles [15] & 92.64 & 0.9452 & 8.13 \\
Daugman [11] & 100 & 0.9999 & 0.08 \\
Previous [18] & 94.91 & 0.9902 & 2.84 \\
Previous [19] & 99.19 & 0.9989 & 0.57 \\
Proposed & 99.60 & 0.9998 & 0.29 \\
Wildes [13] & - & 0.9975 & 1.76 \\
\hline
\end{tabular}
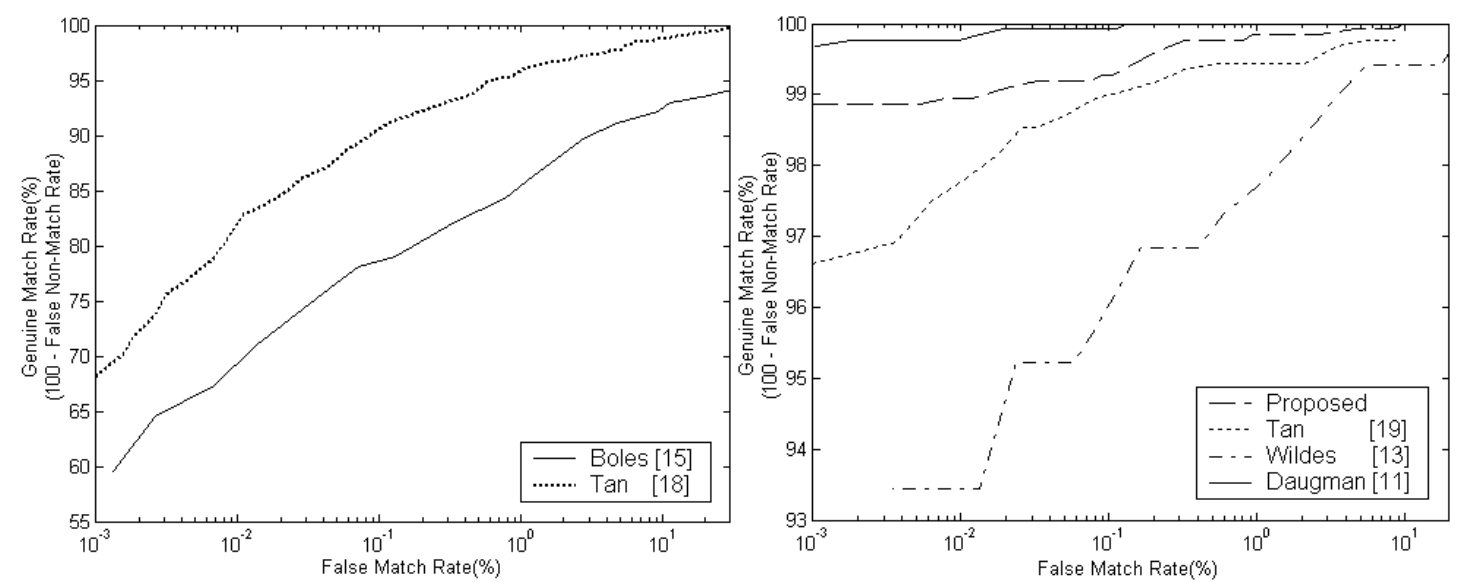

Figure 7. Comparison of ROC curves

Table 4 illustrates that the $\mathrm{Az}$ of each algorithm is greater than 0.9 and the CRR exceeds $90 \%$ as well. This implies the high accuracy of these methods. To better distinguish these well performing methods, the horizontal axis of Figure 7 is spread out using the logarithmic scale. Also, the ROC curves are presented in two plots in order to further improve legibility of Figure 7. From the results shown in Table 4 and Figure 7, we can find that Daugman's method has the best performance, followed by the proposed method and the methods described in [19], [13] (by Wildes et al.), [18] and [15] (by Boles et al.) respectively.

The experimental results demonstrate that our current method is much better than 
our previous methods [18,19] and the method by Wildes et al. [13]. This is determined by their inherent capabilities in representing the image including many randomly distributed characteristics. Generally, the texture-based approach is efficient to process regular features of an image. However, the iris image consists of many randomly distributed and irregular blocks. This implies that texture features may not exactly represent the iris characteristics. The proposed method can effectively analyze local variations of the intensity signals which reflect to a large extent random shape information of the iris and thus achieves much higher performance. Boles and Boashash [15] only employed extremely little information along a virtual circle on the iris to represent the whole iris, which results in a relatively low accuracy as shown in Figure 7. Lim et al. [16] made use of the fourth-level high frequency information of an iris image's 2-D Haar wavelet transform for feature extraction. As we know, the fourth-level details of an image's wavelet decomposition contain essentially very low frequency information. That is, their method did not effectively exploit middle frequency components of the iris which play an important role in recognition as well [11]. Unlike these two methods, our current method employs much more information of the iris and is thus expected to achieve better results. From Table 4 and Figure7, we can see that both Daugman's method and the current method obtain better results than other methods. This is because that they well characterize random shape features of the iris. Binary phase features used in Daugman's algorithm are in essence local shape features of the iris. The proposed iris representation also reflects shape information of the iris by analyzing local variations of the intensity signals. Daugman's method is slightly better than the proposed method in both identification and verification tests. Daugman demodulated phase information of each small local region using multi-scale quadrature wavelets, and then quantized the resulting phasor denoted by a 
complex-valued coefficient to one of the four quadrants in the complex plane. To achieve high accuracy, the size of each local region must be small enough, which results in the high dimensionality of the feature vector (2048 components). That is, his method captures much more information in much smaller local regions. This makes his method slightly better than ours.

Table 5 illustrates the computational cost of the methods described in $[11,13,15,18,19]$ and the current algorithm, including the CPU time for feature extraction (from a preprocessed image to a feature vector) and matching (from a pair of feature vectors to the match result). It should be pointed out that the results only reflect the approximate computational cost of each method since the methods used in experiments are our implementations instead of the original ones.

Table 5. Comparison of the computational complexity

\begin{tabular}{cccc}
\hline Method & $\begin{array}{c}\text { Feature } \\
\text { extraction }(\mathrm{ms})\end{array}$ & $\begin{array}{c}\text { Matching } \\
(\mathrm{ms})\end{array}$ & Others \\
\hline Daugman [11] & 682.5 & 4.3 & - \\
Wildes [13] & 210.0 & 401.0 & Registration \\
Boles [15] & 170.3 & 11.0 & - \\
Previous [18] & 720.3 & 7.5 & - \\
Previous [19] & 426.8 & 13.1 & - \\
Proposed & 260.2 & 8.7 & $\begin{array}{c}\text { Feature } \\
\text { reduction }\end{array}$ \\
\hline
\end{tabular}

The above experiments use 200 different iris images and are carried out in Matlab 6.0 on a $500 \mathrm{Mhz}$ PC with $128 \mathrm{M}$ SDRAM. Since the current method and the method of Boles et al. [15] are based on 1-D signal analysis, they cost less time than others in feature extraction. The method in [13] only takes about $210 \mathrm{~ms}$ to build a four-level Laplacian pyramid representation of an image, whereas the piecewise correlation based matching generally needs high computational expense. Table 5 shows that Daugman's method implements matching faster than others. This is because that this 
method can compute the distance between a pair of feature vectors by the XOR operation. If the XOR operation is performed using some optimization schemes in $\mathrm{C} / \mathrm{C}++$, the running time for matching can be significantly reduced. That is, Daugman's method is efficient to make one-to-many search in a large-scale database. In verification mode (i.e., one-to-one matching), the differences of the computational cost between the above methods (except for the method by Boles et al.) are not very significant. Note that the method in [13] and the proposed method require extra cost for image registration and feature reduction, respectively.

Based on the above results and analysis, we can conclude:

1) The proposed method achieves high performance. This also indicates that local intensity variations are discriminating features for recognition.

2) Compared with texture features (e.g., our previous methods [18,19] and Wildes's method [13]), features based on local intensity variations are more effective for recognition. This is because texture is by nature a regional image property and cannot precisely characterize shape information of the iris.

\subsection{Future work}

The experimental results demonstrate that our attempt to analyze local variations for iris recognition is reasonable and promising. Future work will include characterizing local variations using more effective features as well as representing shape features of the iris using 2-D Gaussian-Hermite moments.

1) Compared with Daugman's method, our current method takes more time for feature matching but has a lower computational cost for feature extraction. We are currently working on exploiting more significant features to represent local variations, for instance, by only computing moments of local sharp variation points as features. This may result in much faster matching speed as well as higher accuracy. In addition, the current scheme for downsampling is only to average the 
adjacent feature elements and does not pay more attention to the most discriminating feature elements for recognition. Therefore, feature selection is an important research issue in the near future.

2) Although the experimental results show that the 1-D signals used in our method well capture the details of the iris, there is an inevitable information loss when transforming the 2-D normalized image into a set of 1-D signals. Therefore, the performance of the current algorithm can be further improved by directly computing the 2-D Gaussian-Hermite moments of the normalized image. Currently, we are also working on how to effectively represent local shapes of the iris using 2-D Gaussian-Hermite moments.

\section{Conclusions}

With the increasing emphasis on security, automated personal identification based on biometrics has been an active topic in pattern recognition. Recently, iris recognition has received increasing attention due to its high reliability. In this paper, we have developed a new iris recognition method which analyzes local variations to characterize the details of the iris. The method first constructs a set of 1-D intensity signals containing the majority of local variations of the iris, and then calculates Gaussian-Hermite moments of such signals as features. To reduce the computational cost and improve the classification accuracy, Fisher linear discriminant and the nearest center classifier are adopted for classification. On the CASIA Iris Database of 2255 images from 213 different subjects, the proposed method achieves encouraging results. We expect to further improve the performance by characterizing local variations using more effective features as well as representing local shapes of the iris using 2-D Gaussian-Hermite moments. 


\section{Acknowledgments}

The authors would like to thank Dr. John Daugman (Cambridge University, UK), Dr. Richard Wildes (York University, Canada) and Dr. Jun Shen (Bordeaux-3 University, France) for their helpful discussions. We also thank the anonymous referees for their thorough reviews and constructive comments. A public version of the CASIA Iris Database is available from http://www.sinobiometrics.com/resources.htm. This work is funded by research grants from the Chinese National Hi-Tech R\&D Program (Grant No. 2001AA114180), the NSFC (Grant No. 69825105) and the CAS. 


\section{References}

[1] A.K. Jain, R.M. Bolle and S. Pankanti, Eds., Biometrics: Personal Identification in a Networked Society, Norwell, MA: Kluwer, 1999.

[2] D. Zhang, Automated Biometrics: Technologies and Systems, Norwell, MA: Kluwer, 2000.

[3] T. Mansfield, G. Kelly, D. Chandler and J. Kane, Biometric Product Testing Final Report, issue 1.0, National Physical Laboratory of UK, 2001.

[4] A. Mansfield and J. Wayman, Best Practice Standards for Testing and Reporting on Biometric Device Performance, National Physical Laboratory of UK, 2002.

[5] F.H. Adler, Physiology of the eye: Clinical Application, Fourth edition, London: The C.V. Mosby Company, 1965.

[6] H. Davision, The Eye, Academic, London, 1962.

[7] R.G. Johnson, "Can Iris Patterns be Used to Identify People? ", Chemical and Laser Sciences Division LA-12331-PR, Los Alamos National Laboratory, Los Alamos, Calif, 1991.

[8] A. Bertillon, "La couleur de l'iris", Rev. Sci., Vol.36, No.3, pp.65-73, 1885.

[9] J.E. Siedlarz, "Iris: More Detailed than a Fingerprint", IEEE Spectrum, Vol.31, pp.27, 1994

[10] J. Daugman, "High Confidence Visual Recognition of Persons by a Test of Statistical Independence", IEEE Trans. on Pattern Analysis and Machine Intelligence, Vol.15, No.11, pp.1148-1161, 1993.

[11] J. Daugman, "Statistical Richness of Visual Phase Information: Update on Recognizing Persons by Iris Patterns", International Journal of Computer Vision, Vol.45, No.1, pp.25-38, 2001.

[12] J. Daugman, "Demodulation by Complex-valued Wavelets for Stochastic Pattern Recognition", International Journal of Wavelets, Multi-resolution and Information Processing, Vol.1, No.1, pp.1-17, 2003.

[13] R. Wildes, J. Asmuth, G. Green, S. Hsu, R. Kolczynski, J. Matey, S. McBride, "A Machine-vision System for Iris Recognition", Machine Vision and Applications, Vol.9, pp.1-8, 1996.

[14] R. Wildes, "Iris Recognition: An Emerging Biometric Technology", Proceedings of the IEEE, Vol.85, pp.1348-1363, 1997.

[15] W. Boles and B. Boashash, "A Human Identification Technique Using Images of the Iris and Wavelet Transform", IEEE Trans. on Signal Processing, Vol.46, No.4, pp.1185-1188, 1998.

[16] S. Lim, K. Lee, O. Byeon and T. Kim, "Efficient Iris Recognition through Improvement of Feature Vector and Classifier", ETRI Journal, Vol.23, No.2, pp.61-70, 2001.

[17] Y. Zhu, T. Tan, Y. Wang, "Biometric Personal Identification Based on Iris Patterns", Proc. of the 15th International Conference on Pattern Recognition, 
Vol.II, pp.805-808, 2000.

[18] L. Ma, Y. Wang, T. Tan, "Iris Recognition Based on Multichannel Gabor Filtering", Proc. of the 5th Asian Conference on Computer Vision, Vol. I, pp.279-283, 2002.

[19] L. Ma, Y. Wang, T. Tan, "Iris Recognition Using Circular Symmetric Filters", Proc. of the 16th International Conference on Pattern Recognition, Vol.II, pp.414-417, 2002.

[20] R. Prokop and A. Reeves, "A Survey of Moment-based Techniques for Unoccluded Object Representation and Recognition", CVGIP: Graphical models and Image Processing, Vol.54, pp.438-460, 1992.

[21] S. Liao, M. Pawlak, "On Image Analysis by Moments", IEEE Trans. on Pattern Analysis and Machine Intelligence, Vol.18, No.3, pp.254-266, 1996.

[22] S. Loncaric, "A Survey of Shape Analysis Techniques", Pattern Recognition, Vol.31, No.8, pp.983-1001, 1998.

[23] J. Shen, "Orthogonal Gaussian-Hermite Moments for Image Characterization", Proc. SPIE, Intelligent Robots and Computer Vision XVI: Algorithms, Techniques, Active Vision, and Materials Handling, pp.224-233, 1997.

[24] J. Shen, W. Shen and D. Shen, "On Geometric and Orthogonal Moments", International Journal of Pattern Recognition and Artificial Intelligence, Vol.14, No.7, pp.875-894, 2000.

[25] D.L. Swets and J.J. Weng, "Using Discriminant Eigenfeatures for Image Retrival”, IEEE Trans. on Pattern Analysis and Machine Intelligence, Vol.18, No.8, pp.831-836, 1996.

[26] P.N. Belhumeur, J.P. Hespanha and D.J. Kriegman, "Eigenfaces vs. Fisherfaces: Recognition Using Class Specific Linear Projection”, IEEE Trans. on Pattern Analysis and Machine Intelligence, Vol.19, No.7, pp.711-720, 1997.

[27] W. Zhao, R. Chellappa and P. Phillips, "Subspace Linear Discriminant Analysis for Face Recognition”, Tech Report CAR-TR-914, Center for Automation Research, University of Maryland, 1999.

[28] C. Liu and H. Wechsler, "Gabor Feature Based Classification Using the Enhanced Fisher Linear Discriminant Model for Face Recognition", IEEE Trans. on Image Processing, Vol.11, No.4, pp.467-476, 2002.

[29] K. Fukunaga, Introduction to Statistical Pattern Recognition, Academic Press, second edition, 1991.

[30] L. Flom and A. Safir, "Iris Recognition System", U.S. Patent, No.4641394, 1987.

[31] J. Daugman, "Biometric Personal Identification System Based on Iris Analysis", U.S. Patent, No.5291560, 1994.

[32] R. Wildes, J. Asmuth, S. Hsu, R. Kolczynski, J. Matey and S. Mcbride, "Automated, Noninvasive Iris Recognition System and Method", U.S. Patent, No.5572596, 1996. 
About the author-Li MA received his B. Sc. and M. Sc. degrees in Automation Engineering from SouthEast University, China, in 1997 and 2000, respectively. Now, he is a $\mathrm{Ph}$. D candidate of National Lab of Pattern Recognition, Institute of Automation, Chinese Academy of Sciences. His current research interests include image processing, pattern recognition and biometrics.

About the author-Tieniu TAN received his B. Sc. in electronic engineering from Xi'an Jiaotong University, China, in 1984 and M. Sc., DIC and Ph. D. in electronic engineering from Imperial College of Science, Technology and Medicine, London, UK, in 1986, 1986, and 1989, respectively.

He joined the Computational Vision Group at the Department of Computer Science, University of Reading, England, in October 1989, where he worked as Research Fellow, Senior Research Fellow and Lecturer. In January 1998, he returned to China to join National Laboratory of Pattern Recognition, Institute of Automation of Chinese Academy of Sciences, Beijing, China. He is currently Professor and Director of the National Laboratory of Pattern Recognition as well as President of the Institute of Automation. He has published widely on image processing, computer vision and pattern recognition. His current research interests include speech and image processing, machine and computer vision, pattern recognition, multimedia, and robotics. He serves as referee for many major national and international journals and conferences. He is an Associate Editor of Pattern Recognition and IEEE Transactions on Pattern Analysis and Machine Intelligence, the Asia Editor of Image and Vision Computing. Dr. Tan was an elected member of the Executive Committee of the British Machine Vision Association and Society for Pattern Recognition (1996-1997) and is a founding co-chair of the IEEE International Workshop on Visual Surveillance. 
About the author-Dexin ZHANG received his B. Sc. degree in Automation Engineering from Tsinghua University, China, in 2000. Now, he is a master student of National Lab of Pattern Recognition, Institute of Automation, Chinese Academy of Sciences. His current research interests include computer vision, pattern recognition and biometrics.

About the author-Yunhong WANG received her B. Sc. degree in Northwestern Polytechnical University, China, in 1989. She earned M. Sc. and Ph. D degrees in Electronic Engineering from Nanjing University of Science and Technology in 1995 and 1998, respectively. Since 1998, she worked at National Lab of Pattern Recognition (NLPR), Institute of Automation, Chinese Academy of Sciences. Now she is an associate professor of the NLPR. Her research interests include biometrics, pattern recognition and image processing. She has published many papers on biometrics and neural networks. 